\title{
Model Splitting and First Order Sliding Mode Observers for Estimation and Diagnosis in Vehicle
}

\author{
B. Jaballah ${ }^{* * *}$ N. K. M'Sirdi ${ }^{*}$ A. Naamane ${ }^{*}$ H. Messaoud ${ }^{* *}$ \\ * LSIS, CNRS UMR 6168, Domaine Univ. St Jerome, Av. Escadrille \\ Normandie-Niemen. 13397, Marseille Cedex 20, France \\ (e-mail:belgacem.jaballah@lsis.org; nacer.msirdi@lsis.org) \\ ** ATSI, Ecole Nationale d'Ingnieurs de Monastir, Rue Ibn El Jazzar, \\ 5019 Monastir, Tunisia (e-mail: Hassani.messaoud@enim.rnu.tn)
}

\begin{abstract}
In this paper a 16 Dof vehicle model is decomposed and used for partial state observation and some inputs estimation. The used sub-models are non linearly coupled and shown to behave well in application. Robust partial state observers with estimation of unknown inputs are developed using First Order Sliding Modes.
\end{abstract}

Keywords: Vehicles Dynamic; Sliding Modes observer ; Robust nonlinear observers; Robust Estimation; Diagnosis and testing; Coupled Sub-Models; Passive Systems.

\section{INTRODUCTION}

In literature, many studies deal with vehicle modeling, but model properties are never detailed nor its passivity emphasized. Vehicle dynamics can be represented by approximate models which are either too much simplified to be realistic or complex and have a variable structure (M'sirdi et al. [2007a]). This kind of systems are composed with many passively coupled subsystems: wheels, motor and braking control system, suspensions, steering, more and more inboard and embedded electronics. There are several non linear parts, which are coupled. These coupling may be time varying and non stationary (M'sirdi et al. [2007c]). Approximations have to be made carefully regarding to the desired application.

In previous works a good nominal vehicle model with 16 DOF have been developed and validated for a French vehicle type (P406) (ElHadri et al. [2000]). Several interesting applications was successful and have been evaluated by use of this model before actual results (M'sirdi et al. [2004], Rabhi et al. [2004]). We have also considered this modeling for estimation of unknown inputs (M'sirdi et al. [2006b]), interaction parameters and exchanges with environment (M'sirdi et al. [2004]). This approach has been used successfully also for heavy vehicles (M'sirdi et al. [2006a]).

In this paper this model is revisited and structured for estimation of inputs and diagnosis. We split the model in five subsystems (M'sirdi et al. [2007b]), regrouped in 3 blocks and then show and justify the rationale behind the successful splitting. The subsystems and the overall system obey the passivity property. This feature, like in Bond Graphs modeling emphasize the energy flow and exchanges between the system parts and also with the

\footnotetext{
* This work was supported by GTAA of GdR MACS (CNRS) and the French Carnot Institute "IC STAR". Acknowledgement is addressed to GII department of POLYTECH Marseille.
}

environment. After the structure and model analysis, we consider estimation of the partial states for diagnosis and motion control in the vehicle. Robust estimations are necessary to be able to obtain good evaluation of the driving situation at each time instant.

\section{NOMINAL VEHICLE MODELING}

Let us consider the fixed reference frame $\mathrm{R}$ and represent the vehicle by the scheme of figure (1) (M'sirdi et al. [2007a], M'sirdi et al. [2007b], M'sirdi et al. [2007c] ). The generalized coordinates vector $q \in R^{16}$ is defined as :

$q^{T}=\left[x, y, z, \theta_{x}, \theta_{y}, \theta_{z}, q_{31}, q_{32}, q_{33}, q_{34}, \delta_{3}, \delta_{4}, \varphi_{1}, \varphi_{2}, \varphi_{3}, \varphi_{4}\right]$ where $x, y$, and $z$ represent displacements. Angles of roll, pitch and yaw are $\theta_{x}, \theta_{y}$ and $\theta_{z}$ respectively. The suspensions elongations are noted $q_{3 i}:(i=1 . .4) . \delta_{i}$ : stands for the steering angles $(i=3,4) . \varphi_{i}$ : are angles of wheels rotations $(i=1 . .4) . \dot{q}, \ddot{q} \in R^{16}$ are respectively velocities and corresponding accelerations.

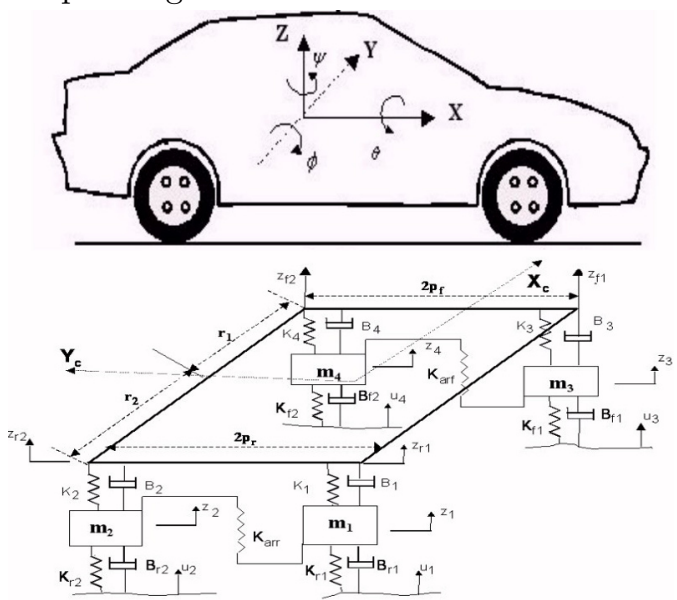

Fig. 1. Vehicle dynamics and reference frames 


\subsection{Global Dynamics Model}

The Nominal model of the vehicle with uncertainties is developed in assuming the car body rigid and pneumatic contact permanent and reduced to one point for each wheel. The vehicle motion can be described by the passive model: (M'sirdi et al. [2004])

$$
\begin{aligned}
\tau & =M(q) \ddot{q}+C(q, \dot{q}) \dot{q}+V(q, \dot{q})+\eta_{o}(t, q, \dot{q}) \\
\tau & =\Gamma_{e}+\Gamma=\Gamma_{e}+J^{T} F
\end{aligned}
$$

The input torque $\tau$ is composed by a part produced by the actuators, which can be assumed to be given by some feedback function and reaction of the road. In equation (2), we remark that there are control inputs from driving and external inputs coming from exchanges with environment (road reactions).

The matrix $M(q)$, of dimension $16 \times 16$, is Symmetric Positive Definite (SPD) inertia matrix and $C(q, \dot{q})$, of dimension too $16 \times 16$, is matrix of coriolis and centrifugal forces.(see details of the two matrix $M(q)$ and $C(q, \dot{q})$ in the appendix A)(ElHadri et al. [2000] and Canudas-deWit et al. [2003])

The vector $V(q, \dot{q})=\xi\left(K_{v} \dot{q}+K_{p} q\right)+G(q)$ are suspensions and gravitation forces (with $K_{v}$ and $K_{p}$ are respectively damping and stiffness matrices, $G(q)$ is the gravity term and $\xi$ is equal to unity when the corresponding wheel is in contact with the ground and zero if not). $\eta_{o}\left(t, x_{1}, x_{2}\right)$ are the uncertainties and neglected dynamics.

The vector $\Gamma_{e}$ represent external inputs for perturbations. The environment reactions are represented by $J^{T} F$ where $J$ is the Jacobian matrix (see detail in appendix A), of dimensions $16 \times 12$, and $F$ is the input forces vector acting on the wheels, it has 12 components (longitudinal $F_{x i}$, lateral $F_{y i}$ and normal $F_{z i}, i=1 . .4$ ): (Canudas-deWit et al. [2003])

$F=\left[F_{x 1}, F_{y 1}, F_{z 1}, F_{x 2}, F_{y 2}, F_{z 2}, F_{x 3}, F_{y 3}, F_{z 3}, F_{x 4}, F_{y 4}, F_{z 4}\right]$

\subsection{Coupled sub models}

We can split the previous model (1) while considering: the inertia matrix as composed by five lines and five column as follows and split positions in five parts by writing $q^{T}=\left[q_{1}^{T}, q_{2}^{T}, q_{3}^{T}, q_{4}^{T}, q_{5}^{T}\right]:$

$$
\begin{aligned}
& q_{1}^{T}=[x, y, z] \\
& q_{2}^{T}=\left[\theta_{x}, \theta_{y}, \theta_{z}\right] \\
& q_{3}^{T}=\left[q_{31}, q_{32}, q_{33}, q_{34}\right] \\
& q_{4}^{T}=\left[\delta_{3}, \delta_{4}\right] \\
& q_{5}^{T}=\left[\varphi_{1}, \varphi_{2}, \varphi_{3}, \varphi_{4}\right]
\end{aligned}
$$

By considering too the vectors $\Gamma_{e}, V(q, \dot{q})$ and $\eta_{o}\left(t, x_{1}, x_{2}\right)$ are splitting as follows :

$$
\begin{gathered}
\Gamma_{e}=\left[\begin{array}{lllll}
0 & 0 & 0 & \Gamma_{e 4} & \Gamma_{e 5}
\end{array}\right]^{T} \\
V(q, \dot{q})=\left[\begin{array}{lllll}
V_{1} & V_{2} & V_{3} & V_{4} & V_{5}
\end{array}\right]^{T} \\
\eta_{o}\left(t, x_{1}, x_{2}\right)=\left[\begin{array}{lllll}
\eta_{1} & \eta_{2} & \eta_{3} & \eta_{4} & \eta_{5}
\end{array}\right]^{T}
\end{gathered}
$$

The 16 Degrees of Freedom model is then equivalent to: (M'sirdi et al. [2008], M'sirdi et al. [2007c])

$$
\begin{aligned}
& {\left[\begin{array}{c}
0 \\
0 \\
0 \\
\Gamma_{e 4} \\
\Gamma_{e 5}
\end{array}\right]+\left[\begin{array}{c}
J_{1}^{T} \\
J_{2}^{T} \\
J_{3}^{T} \\
0 \\
0
\end{array}\right] F=\left[\begin{array}{lllll}
\bar{M}_{11} & \bar{M}_{12} & \bar{M}_{13} & 0 & 0 \\
\bar{M}_{21} & \bar{M}_{22} & \bar{M}_{23} & \bar{M}_{24} & \bar{M}_{25} \\
\bar{M}_{31} & \bar{M}_{32} & \bar{M}_{33} & 0 & 0 \\
0 & \bar{M}_{42} & 0 & \bar{M}_{44} & 0 \\
0 & \bar{M}_{52} & 0 & 0 & \bar{M}_{55}
\end{array}\right]\left[\begin{array}{l}
\ddot{q}_{1} \\
\ddot{q}_{2} \\
\ddot{q}_{3} \\
\ddot{q}_{4} \\
\ddot{q}_{5}
\end{array}\right] } \\
&+ {\left[\begin{array}{lllll}
0 & \bar{C}_{12} & \bar{C}_{13} & 0 & 0 \\
0 & \bar{C}_{22} & \bar{C}_{23} & \bar{C}_{24} & \bar{C}_{25} \\
0 & \bar{C}_{32} & \bar{C}_{33} & 0 & 0 \\
0 & \bar{C}_{42} & 0 & 0 & \bar{C}_{45} \\
0 & \bar{C}_{52} & 0 & \bar{C}_{54} & 0
\end{array}\right]\left[\begin{array}{l}
\dot{q}_{1} \\
\dot{q}_{2} \\
\dot{q}_{3} \\
\dot{q}_{4} \\
\dot{q}_{5}
\end{array}\right]+\left[\begin{array}{l}
V_{1} \\
V_{2} \\
V_{3} \\
V_{4} \\
V_{5}
\end{array}\right]+\left[\begin{array}{l}
\eta_{1} \\
\eta_{2} \\
\eta_{3} \\
\eta_{4} \\
\eta_{5}
\end{array}\right] }
\end{aligned}
$$

The model (1) is then split in 5 equations corresponding respectively to chassis translations, Chassis rotations, Suspensions elongations, wheel steering and wheel rotations, with as positions $q_{1}, q_{2}, q_{3}, q_{4}$ and $q_{5}$. This leads us to the body's translations dynamics described by equation (7):

$F_{T}=J_{1}^{T} F$

$$
=\bar{M}_{11} \ddot{q}_{1}+\bar{M}_{12} \ddot{q}_{2}+\bar{M}_{13} \ddot{q}_{3}+\bar{C}_{12} \dot{q}_{2}+\bar{C}_{13} \dot{q}_{3}+V_{1}+\eta_{1}
$$

Rotations and orientation motions of the body are in (8):

$$
\begin{aligned}
F_{R} & =J_{2}^{T} F \\
& =\bar{M}_{21} \ddot{q}_{1}+\bar{M}_{22} \ddot{q}_{2}+\bar{M}_{23} \ddot{q}_{3}+\bar{M}_{24} \ddot{q}_{4}+\bar{M}_{25} \ddot{q}_{5}+\bar{C}_{22} \dot{q}_{2} \\
& +\bar{C}_{23} \dot{q}_{3}+\bar{C}_{24} \dot{q}_{4}+\bar{C}_{25} \dot{q}_{5}+V_{2}+\eta_{2}
\end{aligned}
$$

The suspension dynamics are in (9):

$$
\begin{aligned}
F_{S} & =J_{3}^{T} F \\
& =\bar{M}_{31} \ddot{q}_{1}+\bar{M}_{32} \ddot{q}_{2}+\bar{M}_{33} \ddot{q}_{3}+\bar{C}_{32} \dot{q}_{2}+\bar{C}_{33} \dot{q}_{3}+V_{3}+\eta_{3}
\end{aligned}
$$

The rest in (10-11) is for wheels steering and rotations:

$$
\begin{aligned}
& \Gamma_{e 4}=\bar{M}_{42} \ddot{q}_{2}+\bar{M}_{44} \ddot{q}_{4}+\bar{C}_{42} \dot{q}_{2}+\bar{C}_{45} \dot{q}_{5}+V_{4}+\eta_{4} \\
& \Gamma_{e 5}=\bar{M}_{52} \ddot{q}_{2}+\bar{M}_{55} \ddot{q}_{5}+\bar{C}_{52} \dot{q}_{2}+\bar{C}_{54} \dot{q}_{4}+V_{5}+\eta_{5}
\end{aligned}
$$

It is worthwhile to note that until now there are no approximations when considering the 5 equations. Approximations will be made when neglecting the coupling terms $\eta_{c}^{i}$. In the previous expressions, we remark that splitting the model is helpful, when using reduced models, to identify what is neglected regard to our proposed nominal model with 16 DoF. $\eta_{c}^{i}$ are coupling terms du to connections with the other sub systems. We can verify that these terms are bounded such and as $\left|\eta_{c}^{i}\right|<k_{i} \forall t, i=(1 . .5)$.

$$
\begin{aligned}
& \Sigma_{11}: \ddot{q}_{1}=f_{1}\left(q_{1}, \dot{q}_{1}, F_{T}\right)+\eta_{c}^{1} \\
& \Sigma_{12}: \ddot{q}_{2}=f_{2}\left(q_{2}, \dot{q}_{2}, F_{R}\right)+\eta_{c}^{2} \\
& \Sigma_{2}: \ddot{q}_{3}=f_{3}\left(q_{3}, \dot{q}_{3}, F_{S}\right)+\eta_{c}^{3} \\
& \Sigma_{31}: \ddot{q}_{4}=f_{4}\left(q_{4}, \dot{q}_{4}, \Gamma_{e 4}\right)+\eta_{c}^{4} \\
& \Sigma_{32}: \ddot{q}_{5}=f_{5}\left(q_{5}, \dot{q}_{5}, \Gamma_{e 5}\right)+\eta_{c}^{5}
\end{aligned}
$$

This can be presented as subsystems $\Sigma_{11}, \Sigma_{12}, \Sigma_{2}, \Sigma_{31}$ and $\Sigma_{32}$ corresponding respectively to chassis translations, rotations, suspensions elongations, wheels steering and rotations. The scheme of figure (2) represents the various blocks of the model that we develop them in the following part. The two subsystems, $\Sigma_{11}$ and $\Sigma_{12}$, represents together the dynamics of the chassis noted, $\Sigma_{1}$. The two subsystems, $\Sigma_{31}$ and $\Sigma_{32}$, us give the wheels dynamics, noted $\Sigma_{3}$.

Dynamics of the chassis $\Sigma_{1}$ : From the global system equations we take the two first equations (7) and (8) for chassis translations and rotations.

$$
\left[\begin{array}{l}
F_{T} \\
F_{R}
\end{array}\right]=\left[\begin{array}{ll}
\bar{M}_{11} & \bar{M}_{12} \\
\bar{M}_{21} & \bar{M}_{22}
\end{array}\right]\left[\begin{array}{l}
\ddot{q}_{1} \\
\ddot{q}_{2}
\end{array}\right]+\left[\begin{array}{l}
\bar{C}_{12} \\
\bar{C}_{22}
\end{array}\right] \dot{q}_{2}+\left[\begin{array}{l}
V_{1} \\
V_{2}
\end{array}\right]+\left[\begin{array}{l}
\eta_{c}^{1} \\
\eta_{c}^{2}
\end{array}\right]
$$

Where the coupling terms, $\eta_{c}^{1}$ and $\eta_{c}^{2}$ are giving by: 


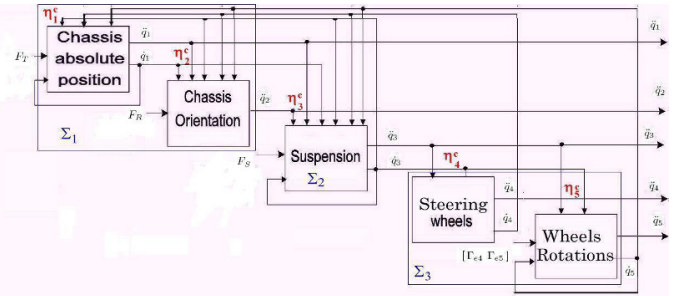

Fig. 2. Five sub models with Coupling terms

$\eta_{c}^{1}=\bar{M}_{13} \ddot{q}_{3}+\bar{C}_{13} \dot{q}_{3}+\eta_{1}$

$\eta_{c}^{2}=\bar{M}_{23} \ddot{q}_{3}+\bar{M}_{24} \ddot{q}_{4}+\bar{M}_{25} \ddot{q}_{5}+\bar{C}_{23} \dot{q}_{3}+\bar{C}_{24} \dot{q}_{4}+\bar{C}_{25} \dot{q}_{5}+\eta_{2}$ By choosing $x_{11}=\left(q_{1}, q_{2}\right)$ and $x_{12}=\left(\dot{q}_{1}, \dot{q}_{2}\right)$, the equivalent state space representation can be written:

$$
\left\{\begin{array}{l}
\dot{x}_{11}=x_{12} \\
\dot{x}_{12}=M_{1}^{-1}\left(J_{12}^{T} F-C_{1} x_{12}-V_{12}-\nu_{1}\right) \\
y_{1}=h\left(x_{11}, x_{12}\right)
\end{array}\right.
$$

Where $M_{1}=\left[\begin{array}{ll}\bar{M}_{11} & \bar{M}_{12} \\ \bar{M}_{21} & \bar{M}_{22}\end{array}\right], C_{1}=\left[\begin{array}{l}\bar{C}_{12} \\ \bar{C}_{22}\end{array}\right], J_{12}^{T}=\left[\begin{array}{c}J_{1}^{T} \\ J_{2}^{T}\end{array}\right]$, $V_{12}=\left[\begin{array}{l}V_{1} \\ V_{2}\end{array}\right]$ and $\mu_{1}=\left[\begin{array}{l}\eta_{c}^{1} \\ \eta_{c}^{2}\end{array}\right]$ are respectively the reduced inertie matrix(SPD), the reduced matrix of Coriolis and Centrifugal forces, the reduced Jacobian matrix and the vector of suspensions and gravitation forces associated to the two subsystems and the coupling term had by dynamics of the other subsystems.

Suspensions Dynamics $\Sigma_{2}$ : We interest now in the dynamics suspensions gives by the third equation (9) from the global one.

$$
F_{S}=\bar{M}_{33} \ddot{q}_{3}+\bar{C}_{33} \dot{q}_{3}+V_{3}+\eta_{c}^{3}
$$

Where $\bar{M}_{33}$ is the inertia matrix associated to subsystem $\Sigma_{2}$, it is SPD. The coupling term $\eta_{c}^{3}$ is giving by:

$$
\eta_{c}^{3}=\bar{M}_{31} \ddot{q}_{1}+\bar{M}_{32} \ddot{q}_{2}+\bar{C}_{32} \dot{q}_{2}+\eta_{3}
$$

Let $x_{2}=\left(x_{21}, x_{22}\right)=\left(q_{3}, \dot{q}_{3}\right)$, the state space representation of the subsystem $\Sigma_{2}$ is then:

$$
\left\{\begin{array}{l}
\dot{x}_{21}=x_{22} \\
\dot{x}_{22}=\bar{M}_{33}^{-1}\left(J_{3}^{T} F-\bar{C}_{33} x_{22}-V_{3}-\mu_{2}\right) \\
y_{2}=h\left(x_{2}\right)
\end{array}\right.
$$

Where $\mu_{2}=\eta_{c}^{3}$

Wheels dynamics $\Sigma_{3}$ : The wheels dynamics are given by the wheels steering of the two front wheels $\left(\Sigma_{31}\right)$, and the wheels rotations of the four wheels $\left(\Sigma_{32}\right)$. Then we can write the fourth equation (10) and the five (11) of the model in the following form:

$$
\begin{aligned}
& \Gamma_{e 4}=\bar{M}_{44} \ddot{q}_{4}+\bar{C}_{45} \dot{q}_{5}+V_{4}+\eta_{c}^{4} \\
& \Gamma_{e 5}=\bar{M}_{55} \ddot{q}_{5}+\bar{C}_{54} \dot{q}_{4}+V_{5}+\eta_{c}^{5}
\end{aligned}
$$

The coupling terms $\eta_{c}^{4}$ and $\eta_{c}^{5}$ of the two subsystem are giving by the following equations:

$$
\begin{aligned}
& \eta_{c}^{4}=\bar{M}_{42} \ddot{q}_{2}+\bar{C}_{42} \dot{q}_{2}+\eta_{4} \\
& \eta_{c}^{5}=\bar{M}_{52} \ddot{q}_{2}+\bar{C}_{52} \dot{q}_{2}+\eta_{5}
\end{aligned}
$$

Then we can write the equations (18 and 19) in the following form:
By choosing $x_{31}=\left(q_{4}, q_{5}\right)$ and $x_{32}=\left(\dot{q}_{4}, \dot{q_{5}}\right)$, the equivalent state space representation can be written:

$$
\left\{\begin{array}{l}
\dot{x}_{31}=x_{32} \\
\dot{x}_{32}=M_{3}^{-1}\left(\Gamma_{e 45}-C_{3} x_{32}-V_{45}-\mu_{3}\right) \\
y_{3}=h\left(x_{31}, x_{32}\right)
\end{array}\right.
$$

Where $M_{3}=\left[\begin{array}{ll}\bar{M}_{44} & 0 \\ 0 & \bar{M}_{55}\end{array}\right], C_{3}=\left[\begin{array}{ll}0 & \bar{C}_{45} \\ \bar{C}_{54} & 0\end{array}\right], \Gamma_{e 45}=$ $\left[\begin{array}{l}\Gamma_{e 4} \\ \Gamma_{e 5}\end{array}\right], V_{45}=\left[\begin{array}{l}V_{4} \\ V_{5}\end{array}\right]$ and $\mu_{3}=\left[\begin{array}{l}\eta_{c}^{4} \\ \eta_{c}^{5}\end{array}\right]$ are respectively the reduced inertie matrix (SPD), the reduced matrix of Coriolis and Centrifugal forces, the reduced external inputs vector and the vector of suspensions and gravitation forces associated to the two subsystems and the coupling term had by dynamics of the other subsystems.

\subsection{Simulation Results}

In order to justify and validate the splitting of the model vehicle applied in the precedent section, we give some simulation results obtained while using a car simulator (SimK106N). The validation of this simulator was made for the laboratory LCPC of Nantes by an instrumented car (peugeot 406). Then we use two steering angle. The figure (3) and (4) represents the various coupling terms by choosing respectively square and sinusoidal steering. We notice that all the coupling terms $\left(\mu_{1}, \mu_{2}, \mu_{3}\right)$ and its squares $\left(\mu_{1}^{2}, \mu_{2}^{2}, \mu_{3}^{2}\right)$ are almost null.
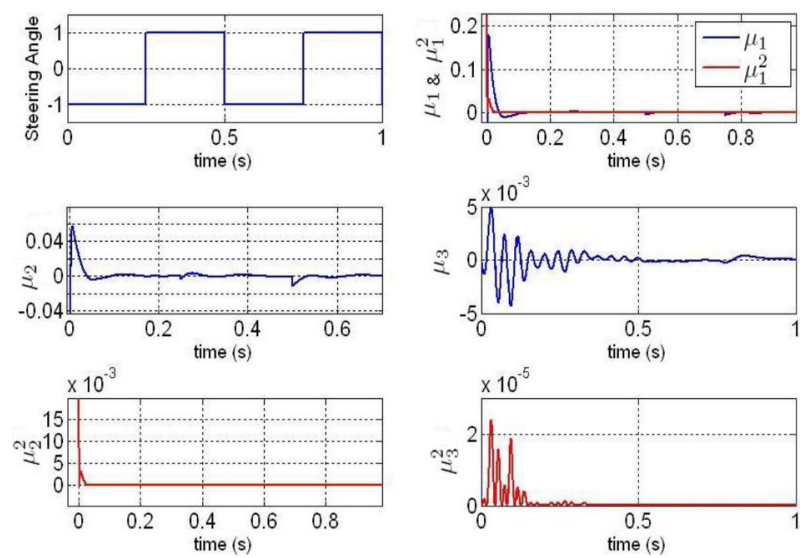

Fig. 3. The Coupling terms for square steering
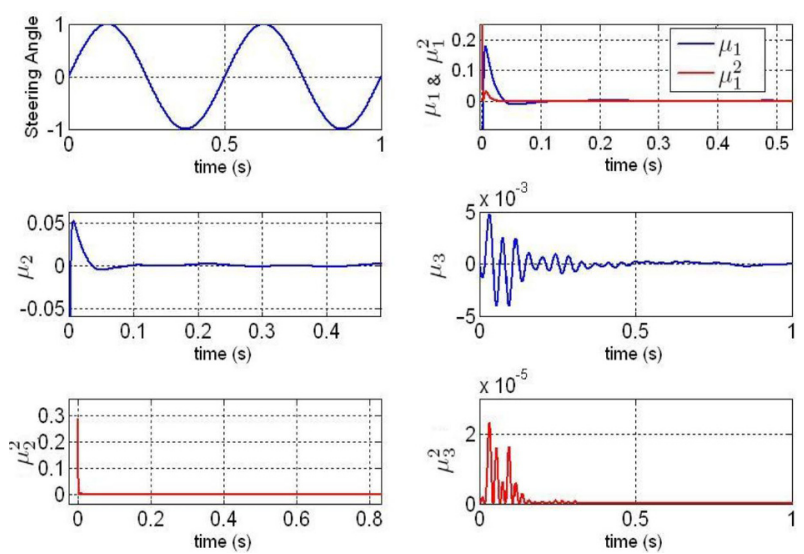

$\left[\begin{array}{l}\Gamma_{e 4} \\ \Gamma_{e 5}\end{array}\right]=\left[\begin{array}{ll}\bar{M}_{44} & 0 \\ 0 & \bar{M}_{55}\end{array}\right]\left[\begin{array}{l}\ddot{q}_{4} \\ \ddot{q}_{5}\end{array}\right]+\left[\begin{array}{ll}0 & \bar{C}_{45} \\ \bar{C}_{54} & 0\end{array}\right]\left[\begin{array}{l}\dot{q}_{4} \\ \dot{q}_{5}\end{array}\right]+\left[\begin{array}{l}V_{4}+\eta_{c}^{4} \\ V_{5}+\eta_{c}^{5}\end{array}\right]$ Fig. 4. The Coupling terms for sinusoidal steering 


\section{FIRST ORDER SLIDING MODE OBSERVERS (FOSM)}

The Sliding mode technique is an attractive approach for robustness (Filippov [1988], Utkin et al. [1999]). In this part we will use a a First Order Sliding Mode (FOSM) to observer the coordinates vector $q_{i}$ for each subsystem and to estimate the input forces vector $F$. To estimate the various coordinates vector $\left(q_{i}\right)$, its derivative $\left(\dot{q}_{i}\right)$ and the forces vector $F$, we propose in this section to develop an observer based on the First Order Sliding Mode approach followed by an estimator. This approach is robust versus the model and the parameters uncertainties for state estimation and is able to reject perturbations and uncertainties effects.

\subsection{Observer for the chassis Dynamics $\Sigma_{1}$ :}

By using the state space representation (14) for the subsystem $\Sigma_{1}$, we propose the following sliding mode observer giving the estimates $\hat{x}_{11}, \hat{x}_{12}$ in two steps:

$$
\left\{\begin{array}{l}
\dot{\hat{x}}_{11}=\hat{x}_{12}-\Lambda_{11} \operatorname{sign}\left(\hat{x}_{11}-x_{11}\right) \\
\dot{\hat{x}}_{12}=M_{1}^{-1}\left(J_{12}^{T} \hat{F}-C_{1} \hat{x}_{12}-V_{12}-\mu_{1}\right)-\Lambda_{12} \operatorname{sign}\left(\tilde{x}_{11}\right) \\
\dot{\hat{F}}=-P_{1} \Lambda_{13} \operatorname{sign}\left(\hat{x}_{11}-x_{11}\right)
\end{array}\right.
$$

Where $P_{1} \in R^{12 \times 12}$ is a positive definite matrix and $\Lambda_{11}$, $\Lambda_{12}, \Lambda_{13}$ are observer gains to be adjusted for convergence, $\hat{F}$ is an a priori estimation of the forces and sign is the vector of $\operatorname{sign}_{i}$ fuction $(i=1 . .6)$. For convergence analysis, we have to express the state estimation error $\left(\tilde{x}_{11}=\hat{x}_{11}-\right.$ $x_{11}$ ) dynamics equation. Then the equations (14), (23) give the observation error dynamics:

$$
\left\{\begin{array}{l}
\dot{\tilde{x}}_{11}=\tilde{x}_{12}-\Lambda_{11} \operatorname{sign}\left(\tilde{x}_{11}\right) \\
\dot{\tilde{x}}_{12}=M_{1}^{-1}\left(J_{12}^{T} \tilde{F}-C_{1} \tilde{x}_{12}\right)-\Lambda_{12} \operatorname{sign}\left(\tilde{x}_{11}\right) \\
\dot{\tilde{F}}=-P_{1} \Lambda_{13} \operatorname{sign}\left(\tilde{x}_{11}\right)
\end{array}\right.
$$

Where $\tilde{x}_{12}=\hat{x}_{12}-x_{12}$ and $\tilde{F}=\hat{F}-F$.

These can be assumed bounded owing to fact that all involved terms are either estimates or come from a passive mechanical part of the system and $\left|\mu_{1}\right|<\kappa_{0} \forall t$. The Lyapunov function $V_{1}=\frac{1}{2} \tilde{x}_{11}^{T} \tilde{x}_{11}$, help to show that the sliding surface $\tilde{x}_{11}=0$ is attractive surface if $\dot{V}_{1}<0$ :

$$
\dot{V}_{1}=\tilde{x}_{11}^{T}\left(\tilde{x}_{12}-\Lambda_{11} \operatorname{sign}\left(\tilde{x}_{11}\right)\right)
$$

If we choose $\Lambda_{11}=\operatorname{diag}\left(\lambda_{11}^{i}\right)$ such as $\left|\tilde{x}_{12}^{i}\right|<\lambda_{11}^{i}$ (for $i=1, . ., 3)$, the convergence in finite time $\left(t_{0}\right)$ for the subsystem state is obtained: $\hat{x}_{11}$ goes to $x_{11}$ in finit time $t_{0}$, so $\dot{\tilde{x}}_{11}=0 \forall t>t_{0}$ Then we obtain a reduced dynamic for the estimation error:

$$
\begin{aligned}
\dot{\tilde{x}}_{12} & =M_{1}^{-1} J_{12}^{T} \tilde{F}-\Lambda_{12} \Lambda_{11}^{-1} \tilde{x}_{12} \\
\dot{\tilde{F}} & =-P_{1} \Lambda_{13} \Lambda_{11}^{-1} \tilde{x}_{12}
\end{aligned}
$$

For the second step of the convergence proof, consider $V_{2}\left(\tilde{x}_{12}, \tilde{F}\right)=\frac{1}{2} \tilde{x}_{12}^{T} \tilde{x}_{12}+\frac{1}{2} \tilde{F}^{T} P_{1}^{-1} \tilde{F}$ then $\dot{V}_{2}\left(\tilde{x}_{12}, \tilde{F}\right)$ becomes if we let $\Lambda_{13}=\left(M_{1}^{-1}\right)^{T} J_{12} \Lambda_{11}$.

$$
\dot{V}_{2}=-\tilde{x}_{12}^{T} \Lambda_{12} \Lambda_{11}^{-1} \tilde{x}_{12}
$$

Now as previously choose $\lambda_{11}^{i}$ and $\lambda_{12}^{i}$ (the diagonal elements of the gain matrices $\Lambda_{11}$ et $\Lambda_{12}$ ) large enough and $\Lambda_{13}=\left(M_{1}^{-1}\right)^{T} J_{12} \Lambda_{11}$. Then convergence of $\left(\hat{x}_{11}, \hat{x}_{21}\right)$ toward $\left(x_{11}, x_{21}\right)$ is obtained and estimation errors on forces are bounded.

\subsection{Observer for Suspensions dynamics $\Sigma_{2}$ :}

We assume that the wheels are always in contact with the ground and note $\tilde{x}_{21}=\hat{x}_{21}-x_{21}, \tilde{x}_{22}=\hat{x}_{22}-x_{22}$ the state estimation error and $\tilde{F}=\hat{F}-F$ force estimation error. The proposed observer, for each wheel suspension, is:

$$
\left\{\begin{array}{l}
\dot{\hat{x}}_{21}=\hat{x}_{22}-\Lambda_{21} \operatorname{sign}\left(\tilde{x}_{21}\right) \\
\dot{\hat{x}}_{22}=\bar{M}_{33}^{-1}\left(J_{3}^{T} \hat{F}-\bar{C}_{33} \hat{x}_{22}-V_{3}-\mu_{2}\right)-\Lambda_{22} \operatorname{sign}\left(\tilde{x}_{21}\right) \\
\dot{\hat{F}}=-P_{2} \Lambda_{23} \operatorname{sign}\left(\tilde{x}_{21}\right)
\end{array}\right.
$$

The observation error dynamics is given by:

$$
\begin{aligned}
\dot{\tilde{x}}_{21} & =\tilde{x}_{22}-\Lambda_{21} \operatorname{sign}\left(\tilde{x}_{21}\right) \\
\dot{\tilde{x}}_{22} & =\bar{M}_{33}^{-1}\left(J_{3}^{T} \tilde{F}-\bar{C}_{33} \tilde{x}_{22}\right)-\Lambda_{22} \operatorname{sign}\left(\tilde{x}_{21}\right) \\
\dot{\tilde{F}} & =-P_{2} \Lambda_{23} \operatorname{sign}\left(\tilde{x}_{21}\right)
\end{aligned}
$$

Like the previous case we choose $V_{1}=\frac{1}{2} \tilde{x}_{21}^{T} \tilde{x}_{21}$ and show that $\hat{x}_{12}$ converges to $x_{12}$ in finite time $t_{02}$ if we ensure that $\forall t>t_{02}$ that $\left|\tilde{x}_{22}\right|<\lambda_{21}^{i}$, (with $\Lambda_{21}=\operatorname{diag}\left(\lambda_{21}^{i}\right)$, $i=1 \ldots 4)$.

Then we deduce the reduced dynamics $\operatorname{sign}_{m o y}\left(\tilde{x}_{21}\right)=$ $\Lambda_{21}^{-1} \tilde{x}_{22}$, if we replace this function by his expression in the equations (31), (32) we obtain:

$$
\begin{aligned}
\dot{\tilde{x}}_{22} & =\bar{M}_{33}^{-1} J_{3}^{T} \tilde{F}-\left(\bar{M}_{33}^{-1} \bar{C}_{33}+\Lambda_{22} \Lambda_{21}^{-1}\right) \tilde{x}_{22} \\
\dot{\tilde{F}} & =-P_{2} \Lambda_{23} \Lambda_{21}^{-1} \tilde{x}_{22}
\end{aligned}
$$

Let $V_{2}=\frac{1}{2} \tilde{x}_{22}^{T} \bar{M}_{33} \tilde{x}_{22}+\frac{1}{2} \tilde{F}^{T} P_{2}^{-1} \tilde{F}$, its derivative $\dot{V}_{2}$ becomes, if we take $\Lambda_{23}=J_{3} \Lambda_{12}$,

$$
\dot{V}_{2}=-\tilde{x}_{22}^{T}\left(\bar{C}_{33}+\bar{M}_{33} \Lambda_{22} \Lambda_{21}^{-1}\right) \tilde{x}_{22}
$$

We can conclude as previously that if we choose $\lambda_{21}$ and $\lambda_{22}$ (the diagonal elements of the gain matrices $\Lambda_{21}$ et $\Lambda_{22}$ ) large enough and $\Lambda_{23}=J_{3} \Lambda_{21}$ then convergence of $\left(\hat{x}_{21}, \hat{x}_{22}\right)$ toward $\left(x_{21}, x_{22}\right)$ is obtained and estimation errors on forces $\tilde{F}$ remains only bounded.

\subsection{Observer for Wheels Dynamics $\Sigma_{3}$ :}

By using the state space representation for the subsystem $\Sigma_{3}$, the proposed observer, for each wheel, is as follows:

$$
\left\{\begin{array}{l}
\dot{\hat{x}}_{31}=\hat{x}_{32}-\Lambda_{31} \operatorname{sign}\left(\hat{x}_{31}-x_{31}\right) \\
\dot{\hat{x}}_{32}=M_{3}^{-1}\left(\Gamma_{e 45}-C_{3} \hat{x}_{32}-V_{45}-\mu_{3}\right)-\Lambda_{32} \operatorname{sign}\left(\tilde{x}_{31}\right) \\
\dot{\hat{F}}=-P_{3} \Lambda_{33} \operatorname{sign}\left(\hat{x}_{31}-x_{31}\right)
\end{array}\right.
$$

The vector $\Gamma_{e 45}$ is assumed known. This observer can be easily extended to estimate the torque by adding an equation defining the drive line producing the torque. Observation error dynamics is then:

$$
\left\{\begin{array}{l}
\dot{\tilde{x}}_{31}=\tilde{x}_{32}-\Lambda_{31} \operatorname{sign}\left(\tilde{x}_{31}\right) \\
\dot{\tilde{x}}_{32}=-M_{3}^{-1} C_{3} \tilde{x}_{32}-\Lambda_{32} \operatorname{sign}\left(\tilde{x}_{31}\right) \\
\dot{\tilde{F}}=-P_{3} \Lambda_{33} \operatorname{sign}\left(\tilde{x}_{31}\right)
\end{array}\right.
$$

with $\tilde{x}_{31}=\hat{x}_{31}-x_{31}, \tilde{x}_{32}=\hat{x}_{32}-x_{32}$ the errors on estimations of states $x_{3}$, and forces $\tilde{F}=\hat{F}-F$. We can prove the convergence in finite time $\left(t_{03}\right)$ of states 
estimates $\hat{x}_{31}$ and bounded of forces estimation by using the Lyapunov functions:

$$
\begin{aligned}
V_{1} & =\frac{1}{2} \tilde{x}_{31} \tilde{x}_{31}^{T} \\
V_{2} & =-\frac{1}{2} \tilde{x}_{32} M_{3} C_{3}^{-1} \tilde{x}_{32}^{T}
\end{aligned}
$$

The derivatives of these functions are:

$$
\begin{aligned}
& \dot{V}_{1}=\tilde{x}_{31}\left(\tilde{x}_{32}-\Lambda_{31} \operatorname{sign}\left(\tilde{x}_{31}\right)\right) \\
& \dot{V}_{2}=-\tilde{x}_{32} C_{3} M_{3}^{-1}\left(-M_{3}^{-1} C_{3} \tilde{x}_{32}-\Lambda_{32} \operatorname{sign}\left(\tilde{x}_{31}\right)\right)
\end{aligned}
$$

To guarantee that these derivatives are negative $\dot{V}_{1}<0$, $\dot{V}_{2}<0$ and consequently the convergence errors $\tilde{x}_{31}$, $\tilde{x}_{32}$ goes to zero in finite time $\left(t_{03}\right)$, we chose $\lambda_{31}^{i}=$ $\operatorname{diag}\left(\Lambda_{31}\right), \lambda_{32}^{i}=\operatorname{diag}\left(\Lambda_{32}\right)$ such as $\lambda_{31}^{i}>\left|\tilde{x}_{32}\right|$ and $\lambda_{32}^{i}>C_{3}^{-1} M_{3}\left|\tilde{x}_{32}\right|$.

\section{SIMULATION RESULTS}

In this section, we give some simulation results got we the simulator previously developed by our staff (SimK106N) in order to test and validate the proposed observers and our approach of model splitting and developing partial state estimators. The system state evolution and forces are computed by use of a car simulation in Matlab Simulink (fig.5). The model of the vehicle which allows

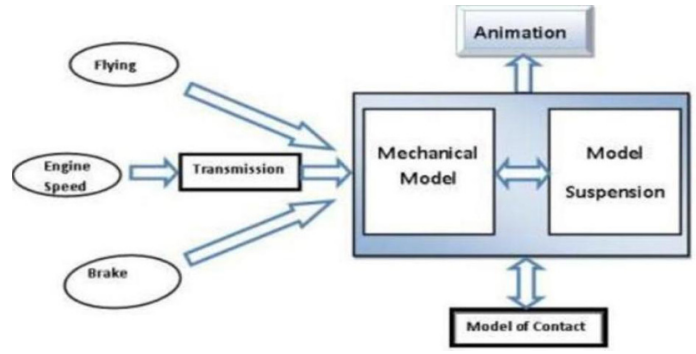

Fig. 5. Modular concept of the simulator

the resolution of the dynamics equation given by (1) is rewritten in following form:

$$
\ddot{q}=M^{-1}(q)\left(\tau-C(q, \dot{q}) \dot{q}-V(q, \dot{q})-\eta_{o}(t, q, \dot{q})\right)
$$

This has been computed with a symbolic toolbox (Maple) and embedded in the following programming structure to give our simulator called SimK106N. The used parameters and environment characteristics have been validated in a previous work in collaboration with the LCPC (ElHadri et al. [2000], M'sirdi et al. [2004], Rabhi et al. [2004]). The

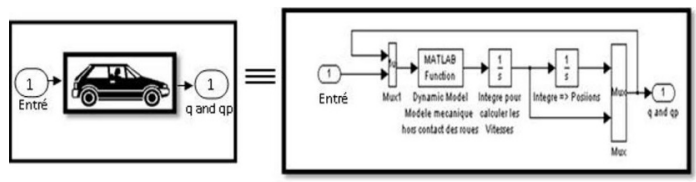

Fig. 6. Simulink block for the resolution of the equations of motion

simulation results presented by the figure (7), is obtained for a driving with sinusoidal steering command of 20 degrees amplitude. The results are good for the First Order Sliding Mode Observers. The model formulation has been done sauch the passivity property is preserved.
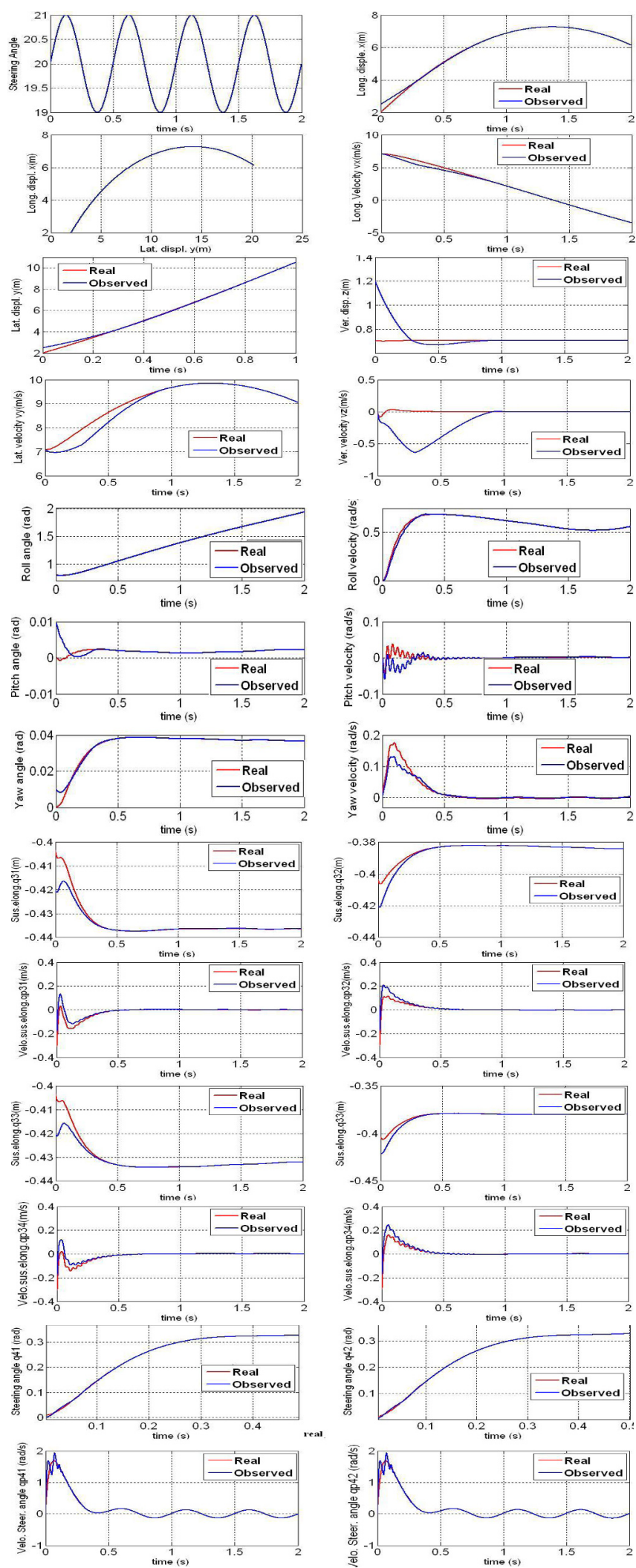

Fig. 7. Results of the First Order Sliding Mode observers

\section{CONCLUSION}

In this paper, we have proposed efficient and robust observers allowing to estimate states and unknown inputs (torques or forces). These observers obey to the first kind assuming that input forces and torques are constant or 
slowly time varying $(\dot{F} \simeq 0)$. The robustness of the sliding mode observer versus uncertainties on model parameters is an important feature. First Order Sliding Mode Observers have been developed and their performance evaluated. These observer are illustrated by simulation results to show effectiveness of their performance. These results validate the proposed observers and our approach of model splitting and developing partial state estimators.

\section{ACKNOWLEDGMENTS}

This work has been done in the context of a the GTAA (Groupe Thématique Automatique et Automobile). The GTAA/GdRMACS is a research group supported by the CNRS.

\section{REFERENCES}

C. Canudas-deWit, P. Tsiotras, E.Velenis, M. Basset, and G.Gissinger. Dynamic friction models for road/tire longitudinal interaction. Vehicle Syst. Dynamics, 2003.

A. ElHadri, G. Beurier, N. K. M'Sirdi, J.C. Cadiou, and Y. Delanne. Simulation et observateurs pour l'estimation des performances dynamiques d'un vehicule. CIFA, 2000.

A. F. Filippov. Differential equations with discontinuous right-hand sides. Dordrecht, The Netherlands:Kluwer Academic Publishers, 1988.

N. K. M'sirdi, A.Rabhi, N.Zbiri, and Y. Delanne. Vrim: Vehicle road interaction modelling for estimation of contact forces. Tyre Colloquium Tyre Models For Vehicle Dynamics Analysis, 2004.

N. K. M'sirdi, A. Boubezoul, A. Rabhi, and L. Fridman. Estimation of performance of heavy vehicles by sliding modes observers. ICINCO:pp360-365, 2006a.

N. K. M'sirdi, A. Rabhi, L. Fridman, J. Davila, and Y. Delanne. Second order sliding mode observer for estimation of velocities, wheel sleep, radius and stiffness. $A C C$, Proceedings of the 2006 American Control Conference Minneapolis,USA,June 14-16:3316-3321, 2006b.

N. K. M'sirdi, A. Rabhi, and A. Naamane. A nominal model for vehicle dynamics and etimation of input forces and tire friction. Conference on control systems, CSC'07, Marrakech, Maroc, 16-18 mai, 2007a.

N. K. M'sirdi, A. Rabhi, and Aziz Naamane. Vehicle models and estimation of contact forces and tire road friction. ICINCO, pages 351-358, 2007b.

N. K. M'sirdi, L. H. Rajaoarisoa, J.F. Balmat, and J. Duplaix. Modelling for control and diagnosis for a class of non linear complex switched systems. Advances in Vehicle Control and Safety AVCS 0\%, Buenos Aires, Argentine, February 8-10, 2007c.

N. K. M'sirdi, B. Jaballah, A. Naamane, and H. Messaoud. Robust observers and unknown input observers for estimation, diagnosis and control of vehicle dynamics. IEEE Inter. Conf. on Intelligent RObots and Systems, IROS08: September 22,Nice, France.http://wwwlasmea.univbpclermont.fr/MEPPC08/, 2008.

A. Rabhi, H. Imine, N. M'Sirdi, and Y. Delanne. Observers with unknown inputs to estimate contact forces and road profile. $A V C S^{\prime} 04$, Inter. Conference on Advances in Vehicle Control and Safety Genova-Italy,October, 2004.

V. Utkin, J. Guldner, and J. Shi. Sliding mode control in electromechanical systems. London, 1999.

\section{Appendix A. PARAMETERS OF THE MODELS}

Definition of the matrices involved in the model.

The inertia matrix: $M=\left[\begin{array}{llllll}\bar{M}_{11} & \bar{M}_{12} & \bar{M}_{13} & 0 & 0 \\ \bar{M}_{21} & M_{22} & \bar{M}_{23} & \bar{M}_{24} & \bar{M}_{25} \\ \bar{M}_{31} & \bar{M}_{32} & M_{33} & 0 & 0 \\ 0 & \bar{M}_{42} & 0 & \bar{M}_{44} & 0 \\ 0 & \bar{M}_{52} & 0 & 0 & \bar{M}_{55}\end{array}\right]$

$\bar{M}_{11}=\left[\begin{array}{lll}M_{1}^{1} & 0 & 0 \\ 0 & M_{2}^{2} & 0 \\ 0 & 0 & M_{3^{3}}\end{array}\right] \bar{M}_{12}=\bar{M}_{21}^{T}=\left[\begin{array}{lll}M_{1}^{4} & M_{1}^{5} & M_{1}^{6} \\ M_{2}^{4} & M_{3}^{5} & M_{2}^{6} \\ 0 & M_{3}^{5} & M_{3}^{6}\end{array}\right]$

$\bar{M}_{13}=\bar{M}_{31}^{T}=\left[\begin{array}{llll}M_{1}^{7} & M_{1}^{8} & M_{1}^{9} & M_{1}^{10} \\ M_{2}^{7} & M_{2}^{8} & M_{2}^{9} & M_{2}^{10} \\ M_{3}^{7} & M_{3}^{8} & M_{3}^{9} & M_{3}^{10}\end{array}\right] \bar{M}_{44}=\left[\begin{array}{lll}M_{11}^{11} & 0 \\ 0 & M_{12}^{12}\end{array}\right]$

$\bar{M}_{23}=\bar{M}_{32}^{T}=\left[\begin{array}{cccc}M_{4}^{7} & M_{4}^{8} & M_{4}^{9} & M_{4}^{10} \\ M_{5}^{7} & M_{5}^{8} & M_{5}^{9} & M_{5}^{10} \\ M_{6}^{7} & M_{6}^{8} & M_{6}^{9} & M_{6}^{10}\end{array}\right]$

$\bar{M}_{24}=\bar{M}_{42}^{T}=\left[\begin{array}{ll}M_{4}^{11} & M_{4}^{12} \\ M_{5}^{11} & M_{5}^{12} \\ 0 & 0\end{array}\right] \bar{M}_{33}=\left[\begin{array}{llll}M_{7}^{7} & 0 & 0 & 0 \\ 0 & M_{8}^{8} & 0 & 0 \\ 0 & 0 & M_{9}^{9} & 0 \\ 0 & 0 & 0 & M_{10}^{10}\end{array}\right]$

$\bar{M}_{25}=\bar{M}_{52}^{T}=\left[\begin{array}{llll}M_{4}^{13} & M_{4}^{14} & M_{4}^{15} & M_{4}^{16} \\ M_{5}^{13} & M_{5}^{14} & M_{5}^{15} & M_{5}^{16} \\ 0 & 0 & M_{6}^{15} & M_{6}^{16}\end{array}\right]$

$\bar{M}_{22}=\left[\begin{array}{lll}M_{4}^{4} & M_{4}^{5} & M_{4}^{6} \\ M_{5}^{4} & M_{5}^{5} & M_{5}^{6} \\ M_{6}^{4} & M_{6}^{5} & M_{6}^{6}\end{array}\right] \bar{M}_{55}=\left[\begin{array}{llll}M_{13}^{13} & 0 & 0 & 0 \\ 0 & M_{14}^{14} & 0 & 0 \\ 0 & 0 & M_{15}^{15} & 0 \\ 0 & 0 & 0 & M_{16}^{16}\end{array}\right]$

The coriolis and centrifugal matrix $C$ :

$$
\begin{aligned}
& C=\left[\begin{array}{lllll}
0 & \bar{C}_{12} & \bar{C}_{13} & 0 & 0 \\
0 & \bar{C}_{22} & \bar{C}_{23} & \bar{C}_{24} & \bar{C}_{25} \\
0 & \bar{C}_{32} & 0 & 0 & 0 \\
0 & \bar{C}_{42} & 0 & 0 & \bar{C}_{45} \\
0 & \bar{C}_{52} & 0 & \bar{C}_{54} & 0
\end{array}\right] ; \bar{C}_{12}=\left[\begin{array}{lll}
C_{14} & C_{15} & C_{16} \\
C_{24} & C_{25} & C_{26} \\
0 & C_{35} & C_{36}
\end{array}\right] ; \\
& \bar{C}_{13}=\left[\begin{array}{llll}
C_{17} & C_{18} & C_{19} & C_{110} \\
C_{27} & C_{28} & C_{29} & C_{210} \\
C_{37} & C_{38} & C_{39} & C_{310}
\end{array}\right] ; \bar{C}_{22}=\left[\begin{array}{lll}
C_{44} & C_{45} & C_{46} \\
C_{54} & C_{55} & C_{56} \\
C_{64} & C_{65} & C_{66}
\end{array}\right] ; \\
& \bar{C}_{24}=\bar{C}_{42}^{T}=\left[\begin{array}{ll}
C_{411} & C_{412} \\
C_{511} & C_{512} \\
C_{611} & C_{612}
\end{array}\right] ; \bar{C}_{45}=\left[\begin{array}{llll}
0 & 0 & C_{1115} & 0 \\
0 & 0 & 0 & C_{1216}
\end{array}\right] \\
& \bar{C}_{23}=\bar{C}_{32}^{T}=\left[\begin{array}{llll}
C_{47} & C_{48} & C_{49} & C_{410} \\
C_{57} & C_{58} & C_{59} & C_{510} \\
C_{67} & C_{68} & C_{69} & C_{610}
\end{array}\right] \text {; } \\
& \bar{C}_{25}=\bar{C}_{52}^{T}=\left[\begin{array}{llll}
C_{413} & C_{414} & C_{415} & C_{416} \\
C_{513} & C_{514} & C_{515} & C_{516} \\
C_{613} & C_{614} & C_{615} & C_{616}
\end{array}\right]
\end{aligned}
$$

The Jacobian matrix: $J=\left[J_{1}^{T} J_{2}^{T} J_{3}^{T} 00\right]_{12 \times 16}^{T}$

$$
\begin{aligned}
& J_{1}^{T}=\left[\begin{array}{llllllllllll}
J_{1}^{1} & 0 & 0 & J_{4}^{1} & 0 & 0 & J_{7}^{1} & 0 & 0 & J_{10}^{1} & 0 & 0 \\
0 & J_{2}^{2} & 0 & 0 & J_{5}^{2} & 0 & 0 & J_{8}^{2} & 0 & 0 & J_{11}^{2} & 0 \\
0 & 0 & J_{3}^{3} & 0 & 0 & J_{6}^{3} & 0 & 0 & J_{9}^{3} & 0 & 0 & J_{12}^{3}
\end{array}\right] \\
& J_{2}^{T}=\left[\begin{array}{lllllllllllll}
J_{1}^{4} & J_{2}^{4} & 0 & J_{4}^{4} & J_{5}^{4} & 0 & J_{7}^{4} & J_{8}^{4} & 0 & J_{10}^{4} & J_{11}^{4} & 0 \\
J_{1}^{5} & J_{2}^{5} & J_{3}^{5} & J_{4}^{5} & J_{5}^{5} & J_{6}^{5} & J_{7}^{5} & J_{8}^{5} & J_{9}^{5} & J_{10}^{5} & J_{11}^{5} & J_{12}^{5} \\
J_{1}^{6} & J_{2}^{6} & J_{3}^{6} & J_{4}^{6} & J_{5}^{6} & J_{6}^{6} & J_{7}^{6} & J_{8}^{6} & J_{9}^{6} & J_{10}^{6} & J_{11}^{6} & J_{12}^{6}
\end{array}\right] \\
& J_{3}^{T}=\left[\begin{array}{llllllllllllll}
J_{1}^{7} & J_{2}^{7} & J_{3}^{7} & 0 & 0 & 0 & 0 & 0 & 0 & 0 & 0 & 0 \\
0 & 0 & 0 & J_{4}^{8} & J_{5}^{8} & J_{6}^{8} & 0 & 0 & 0 & 0 & 0 & 0 \\
0 & 0 & 0 & 0 & 0 & 0 & J_{7}^{9} & J_{8}^{9} & J_{9}^{9} & 0 & 0 & 0 \\
0 & 0 & 0 & 0 & 0 & 0 & 0 & 0 & 0 & J_{10}^{10} & J_{11}^{10} & J_{12}^{10}
\end{array}\right]
\end{aligned}
$$

